\title{
Geraghty-type theorems in modular metric spaces with an application to partial differential equation
}

\author{
Parin Chaipunya', Yeol Je Cho ${ }^{2^{*}}$ and Poom Kumam ${ }^{1 *}$
}

\footnotetext{
* Correspondence: yjcho@gnu.ac.kr; poom.kum@kmutt.ac.th

${ }^{1}$ Department of Mathematics, Faculty of Science, King Mongkut's University of Technology Thonburi, Bangkok 10140, Thailand ${ }^{2}$ Department of Mathematics Education and the RINS, Gyeongsang National University, Chinju 660-701, Korea Full list of author information is available at the end of the article
}

\begin{abstract}
In this article, we prove some fixed point theorems of Geraghty-type concerning the existence and uniqueness of fixed points under the setting of modular metric spaces. Also, we give an application of our main results to establish the existence and uniqueness of a solution to a nonhomogeneous linear parabolic partial differential equation in the last section.

Mathematics Subject Classification (2010): 47H10, 54H25, 35K15.

Keywords: modular metric space, ordered set, Geraghty's theorem, initial value problems, parabolic equation.
\end{abstract}

\section{Introduction and preliminaries}

Throughout this article, let $\mathbb{R}^{+}$denote the set of all positive real numbers and let $\mathbb{R}_{+}$ denote the set of all nonnegative real numbers.

Since the year 1922, Banach's contraction principle, due to its simplicity and applicability, has became a very popular tool in modern analysis, especially in nonlinear analysis including its applications to differential and integral equations, variational inequality theory, complementarity problems, equilibrium problems, minimization problems and many others. Also, many authors have improved, extended and generalized this contraction principle in several ways (see e.g. [1-10]).

In 1973, Geraghty [11] gave an interesting generalization of the contraction principle using the class $\mathcal{S}$ of the functions $\beta: \mathbb{R}_{+} \rightarrow[0,1)$ satisfying the following condition:

$$
\beta\left(t_{n}\right) \rightarrow 1 \text { implies } t_{n} \rightarrow 0 \text {. }
$$

Theorem 1.1. [11] Let $(X, d)$ be a complete metric space and $f$ be a self-mapping on $X$ such that there exists $\beta \in \mathcal{S}$ satisfying

$$
d(f x, f y) \leq \beta(d(x, y)) d(x, y)
$$

for all $x, y \in X$. Then the sequence $\left\{x_{n}\right\}$ defined by $x_{n}=f x_{n-1}$ for each $n \geq 1$ converges to the unique fixed point of $f$ in $X$.

Later, Amini-Harandini et al. [12] extended Geraghty's fixed point theorem to the setting of partially ordered metric spaces as follows:

C 2012 Chaipunya et al; licensee Springer. This is an Open Access article distributed under the terms of the Creative Commons Attribution License (http://creativecommons.org/licenses/by/2.0), which permits unrestricted use, distribution, and reproduction in any medium, provided the original work is properly cited. 
Theorem 1.2. [12] Let $(X$, ㄷ) be a partially ordered metric set and suppose that there exists a metric $d$ in $X$ such that $(X, d)$ is a complete metric space. Let $f$ be a nondecreasing self-mapping on $X$ which satisfies the inequality (1.1) whenever $x, y \in X$ are comparable. Assume that $f$ is either continuous or

if a nondecreasing sequence $\left\{x_{n}\right\}$ converges to $x_{*}$, then $x_{n} \sqsubseteq x_{*}$ for each $n \geq 1$.

If, additionally, the following condition is satisfied:

for any $x, y \in X$, there exists $z \in X$ which is comparable to both $x$ and $y$,

then the sequence $\left\{x_{n}\right\}$ converges to the unique fixed point of $f$ in $X$.

Let $\Psi$ denote the class of functions $\psi: \mathbb{R}_{+} \rightarrow \mathbb{R}_{+}$satisfying the following conditions:

(a) $\psi$ is nondecreasing.

(b) $\psi$ is continuous.

(c) $\psi(t)=0$ if and only if $t=0$.

Using this class, Eshaghi Gordji et al. [13] extended the Theorem 1.2 as follows:

Theorem 1.3. [13] Let $(X, \subseteq)$ be a partially ordered metric set and suppose that there exists a metric $d$ in $X$ such that $(X, d)$ is a complete metric space. Let $f$ be a nondecreasing self-mapping on $X$ such that there exists $x_{0} \in X$ with $x_{0} \subseteq f x_{0}$. Suppose that there exist $\beta \in \mathcal{S}$ and $\psi \in \Psi$ such that

$$
\psi(d(f x, f y)) \leq \beta(\psi(d(x, y))) \psi(d(x, y)),
$$

whenever $x, y \in X$ are comparable. Assume also that the condition (1.2) holds. Then $f$ has a fixed point.

On the other hand, in 2010, Chistyakov [14] introduced the notion of a modular metric space which is raised in an attempt to avoid some restrictions of the concept of a modular space (for the literature of a modular space, see e.g. [15-21] and references therein). Some of the early investigations on metric fixed point theory in this space refer to [22-24].

For the rest of this section, we present some notions and basic facts of modular metric spaces.

Definition 1.4. [14] Let $X$ be a nonempty set. A function $\omega: \mathbb{R}^{+} \times X \times X \rightarrow \mathbb{R}_{+} \cup\{\infty\}$ is said to be a metric modular on $X$ if, for all $x, y, z \in X$, the following conditions hold:

(a) $\omega_{\lambda}(x, y)=0$ for all $\lambda>0$ if and only if $x=y$.

(b) $\omega_{\lambda}(x, y)=\omega_{\lambda}(y, x)$ for all $\lambda>0$.

(c) $\omega_{\lambda+\mu}(x, y) \leq \omega_{\lambda}(x, z)+\omega_{\mu}(z, y)$ for all $\lambda, \mu>0$.

For any $x_{\iota} \in X$, the set $X_{\omega}\left(x_{\iota}\right)=\left\{x \in X: \lim _{\lambda \rightarrow \infty} \omega_{\lambda}\left(x, x_{\iota}\right)=0\right\}$ is called a modular metric space generated by $x_{\iota}$ and induced by $\omega$. If its generator $x_{\iota}$ does not play any role in the situation (that is, $X_{\omega}$ is independent of generators), we write $X_{\omega}$ instead of $X_{\omega}\left(x_{t}\right)$.

Observe that a metric modular $\omega$ on $X$ is nonincreasing with respect to $\lambda>0$. We can simply show this assertion using the condition (c). For any $x, y \in X$ and $0<\mu<\lambda$, we have 


$$
\omega_{\lambda}(x, y) \leq \omega_{\lambda-\mu}(x, x)+\omega_{\mu}(x, y)=\omega_{\mu}(x, y) .
$$

For any $x, y \in X$ and $\lambda>0$, we set

$$
\omega_{\lambda^{+}}(x, y):=\lim _{\epsilon \downarrow 0} \omega_{\lambda+\epsilon}(x, y), \quad \omega_{\lambda-}(x, y):=\lim _{\epsilon \downarrow 0} \omega_{\lambda-\epsilon}(x, y) .
$$

Consequently, from (1.4), it follows that

$$
\omega_{\lambda^{+}}(x, y) \leq \omega_{\lambda}(x, y) \leq \omega_{\lambda^{-}}(x, y) .
$$

For any $x, y \in X$, if a metric modular $\omega$ on $X$ possesses a finite value and $\omega_{\lambda}(x, y)=$ $\omega_{\mu}(x, y)$ for all $\lambda, \mu>0$, then $d(x, y):=\omega_{\lambda}(x, y)$ is a metric on $X$.

Later, Chaipunya et al. [23] has altered the notion of convergent and Cauchy sequences in modular metric spaces under the direction of Mongkolkeha et al. [24].

Definition 1.5. $[23,24]$ Let $X_{\omega}$ be a modular metric space and $\left\{x_{n}\right\}$ be a sequence in $X_{\omega}$.

(1) A point $x \in X_{\omega}$ is called a limit of $\left\{x_{n}\right\}$ if, for each $\lambda, \epsilon>0$, there exists $n_{0} \in \mathbb{N}$ such that $\omega_{\lambda}\left(x_{n}, x\right)<\epsilon$ for all $n \geq n_{0}$. A sequence that has a limit is said to be convergent (or converges to $x$ ), which is written as $\lim _{n \rightarrow \infty} x_{n}=x$.

(2) A sequence $\left\{x_{n}\right\}$ in $X_{\omega}$ is said to be a Cauchy sequence if, for each $\lambda, \epsilon>0$, there exists $n_{0} \in \mathbb{N}$ such that $\omega_{\lambda}\left(x_{n}, x_{m}\right)<\epsilon$ for all $m, n \geq n_{0}$.

(3) If every Cauchy sequences in $X$ converges, $X$ is said to be complete.

In this article, we prove a generalization of Geraghty's theorem which also improves the result of Eshagi Gordji et al. [13] under the influence of a modular metric space. An application to partial differential equation is also provided.

\section{Main results}

Before stating our main results, we first introduce the following classes for a more convenience of usage.

For each $n \in \mathbb{N}$, let $\mathcal{S}_{n}$ denote the class of $n$-tuples of functions $\left(\beta_{1}, \beta_{2}, \ldots, \beta_{n}\right)$, where for each $i \in\{1,2, \ldots, n\}, \beta_{i}: \mathbb{R}_{+} \cup\{\infty\} \rightarrow[0,1)$ and the following implication holds:

$$
\beta\left(t_{k}\right):=\beta_{1}\left(t_{k}\right)+\beta_{2}\left(t_{k}\right)+\cdots+\beta_{n}\left(t_{k}\right) \rightarrow 1 \text { implies } t_{k} \rightarrow 0 .
$$

Actually, Geraghty's class $\mathcal{S}$ is equivalent to the class $\mathcal{S}_{1}$ when $\infty$ is not considered. It follows that, for each $m \in\{1,2, \ldots, n\}$, if $\left(\beta_{1}, \beta_{2}, \ldots, \beta_{m}\right) \in \mathcal{S}_{m}$, then $(\beta_{1}, \beta_{2}, \ldots, \beta_{m}, \underbrace{\theta, \theta, \ldots, \theta}_{n-m \text { entries }}) \in \mathcal{S}_{n}$, where $\theta$ denotes the zero function. Also, note that, if $\underbrace{(\beta, \beta, \ldots, \beta)}_{n \text { entries }} \in \mathcal{S}_{n}$, then we also have the following:

$$
\beta\left(t_{k}\right) \rightarrow \frac{1}{n} \text { implies } t_{k} \rightarrow 0
$$

Besides, if $\left(\beta_{1}, \beta_{2} \ldots, \beta_{n}\right) \in \mathcal{S}_{n}$, then $\pi\left(\left(\beta_{1}, \beta_{2} \ldots, \beta_{n}\right)\right) \in \mathcal{S}_{n}$, where $\pi\left(\left(\beta_{1}, \beta_{2} \ldots, \beta_{n}\right)\right)$ is a permutation of $\left(\beta_{1}, \beta_{2} \ldots, \beta_{n}\right)$. It is also important to know that, if $\left(\beta_{1}, \beta_{2} \ldots, \beta_{n}\right) \in \mathcal{S}_{n}$, then $\left(\beta_{n_{1}}, \beta_{n_{2}}, \ldots, \beta_{n_{m}}\right) \in \mathcal{S}_{m}$ for each $m \in\{1,2, \ldots, n\}$, where each $\beta_{n_{i}}$ is selected from $\left\{\beta_{1}, \beta_{2}, \ldots, \beta_{n}\right\}$ and $\beta_{n_{i}} \neq \beta_{n_{j}}$ for all $i, j \in\{1,2, \ldots, m\}$. 
Let $\bar{\Psi}$ denote the class of functions $\psi: \mathbb{R}_{+} \cup\{\infty\} \rightarrow \mathbb{R}_{+} \cup\{\infty\}$ satisfying the following conditions:

(a) If $0<t<\infty$, then $\psi(t)<\infty$.

(b) $\left.\psi\right|_{\mathbb{R}_{+}} \in \Psi$.

Now, we are ready to give our main results in this article.

Theorem 2.1. Let $X_{\omega}$ be a complete modular metric space with a partial ordering 드 and $f$ be a self-mapping on $X_{\omega}$ such that, for each $\lambda>0$, there exists $\eta(\lambda) \in(0, \lambda)$ such that

$$
\begin{aligned}
\psi\left(\omega_{\lambda}(f x, f y)\right) \leq \alpha & \left(\psi\left(\omega_{\lambda}(x, y)\right)\right) \psi\left(\omega_{\lambda+\eta(\lambda)}(x, y)\right)+\beta\left(\psi\left(\omega_{\lambda}(x, y)\right)\right) \psi\left(\omega_{\lambda}(x, f x)\right) \\
& +\gamma\left(\psi\left(\omega_{\lambda}(x, y)\right)\right) \psi\left(\omega_{\lambda}(y, f y)\right)
\end{aligned}
$$

where $\psi \in \bar{\Psi}$ and $(\alpha, \beta, \gamma) \in \mathcal{S}_{3}$ with $\alpha(t)+2 \max \left\{\sup _{t \geq 0} \beta(t)\right.$, $\left.\sup _{t \geq 0} \gamma(t)\right\}<1$. Assume also that the condition (1.2) holds. If there exists $x_{0} \in X_{\omega}$ such that $\omega_{\lambda}\left(x_{0}, f x_{0}\right)$ $<\infty$ for all $\lambda>0$, then the following hold:

(1) $f$ has a fixed point $x_{\infty} \in X_{\omega}$.

(2) The sequence $\left\{f^{n} x_{0}\right\}$ converges to $x_{\infty}$.

Proof. It is clear that the sequence $\left\{f^{n} x_{0}\right\}$ is nondecreasing. Assume that, for each $n \geq 1$, there exists $\lambda_{n}>0$ such that $\omega_{\lambda_{n}}\left(f^{n} x_{0}, f^{n+1} x_{0}\right) \neq 0$. Otherwise, the proof is complete. For each $n \geq 1$, if $0<\lambda \leq \lambda_{n}$, then we also have $\omega_{\lambda}\left(f^{n} x_{0}, f^{n+1} x_{0}\right) \neq 0$. Since $f^{n} x_{0} \sqsubseteq f^{n+1} x_{0}$, for any $0<\lambda \leq \lambda_{n}$, we have

$$
\begin{aligned}
\psi\left(\omega_{\lambda_{n}}\left(f^{n} x_{0}, f^{n+1} x_{0}\right)\right) \leq & \psi\left(\omega_{\lambda}\left(f^{n} x_{0}, f^{n+1} x_{0}\right)\right) \\
\leq & \alpha\left(\psi\left(\omega_{\lambda}\left(f^{n-1} x_{0}, f^{n} x_{0}\right)\right)\right) \psi\left(\omega_{\lambda+\eta}(\lambda)\left(f^{n-1} x_{0}, f^{n} x_{0}\right)\right) \\
& +\beta\left(\psi\left(\omega_{\lambda}\left(f^{n-1} x_{0}, f^{n} x_{0}\right)\right)\right) \psi\left(\omega_{\lambda}\left(f^{n-1} x_{0}, f^{n} x_{0}\right)\right) \\
& +\gamma\left(\psi\left(\omega_{\lambda}\left(f^{n-1} x_{0}, f^{n} x_{0}\right)\right)\right) \psi\left(\omega_{\lambda}\left(f^{n} x_{0}, f^{n+1} x_{0}\right)\right) \\
\leq & \alpha\left(\psi\left(\omega_{\lambda}\left(f^{n-1} x_{0}, f^{n} x_{0}\right)\right)\right) \psi\left(\omega_{\lambda}\left(f^{n-1} x_{0}, f^{n} x_{0}\right)\right) \\
& +\beta\left(\psi\left(\omega_{\lambda}\left(f^{n-1} x_{0}, f^{n} x_{0}\right)\right)\right) \psi\left(\omega_{\lambda}\left(f^{n-1} x_{0}, f^{n} x_{0}\right)\right) \\
& +\gamma\left(\psi\left(\omega_{\lambda}\left(f^{n-1} x_{0}, f^{n} x_{0}\right)\right)\right) \psi\left(\omega_{\lambda}\left(f^{n} x_{0}, f^{n+1} x_{0}\right)\right),
\end{aligned}
$$

which implies that

$$
\begin{aligned}
\psi\left(\omega_{\lambda}\left(f^{n} x_{0}, f^{n+1} x_{0}\right)\right) & \leq \frac{\alpha\left(\psi\left(\omega_{\lambda}\left(f^{n-1} x_{0}, f^{n} x_{0}\right)\right)\right)+\beta\left(\psi\left(\omega_{\lambda}\left(f^{n-1} x_{0}, f^{n} x_{0}\right)\right)\right)}{1-\gamma\left(\psi\left(\omega_{\lambda}\left(f^{n-1} x_{0}, f^{n} x_{0}\right)\right)\right)} \psi\left(\omega_{\lambda}\left(f^{n-1} x_{0}, f^{n} x_{0}\right)\right) \\
& \leq \psi\left(\omega_{\lambda}\left(f^{n-1} x_{0}, f^{n} x_{0}\right)\right) \\
& \vdots \\
& \leq \psi\left(\omega_{\lambda}\left(x_{0}, f x_{0}\right)\right. \\
& <\infty
\end{aligned}
$$

Therefore, $\left\{\psi\left(\omega_{\lambda}\left(f^{n} x_{0}, f^{n+1} x_{0}\right)\right)\right\}$ is nonincreasing and bounded below. So, the sequence converges to some number $r \geq 0$. Assume $r>0$. Observe that 


$$
\begin{aligned}
\psi\left(\omega_{\lambda}\left(f^{n} x_{0}, f^{n+1} x_{0}\right)\right) \leq & {\left[\alpha\left(\psi\left(\omega_{\lambda}\left(f^{n-1} x_{0}, f^{n} x_{0}\right)\right)\right)+\beta\left(\psi\left(\omega_{\lambda}\left(f^{n-1} x_{0}, f^{n} x_{0}\right)\right)\right)\right.} \\
& \left.+\gamma\left(\psi\left(\omega_{\lambda}\left(f^{n-1} x_{0}, f^{n} x_{0}\right)\right)\right)\right] \psi\left(\omega_{\lambda}\left(f^{n-1} x_{0}, f^{n} x_{0}\right)\right) .
\end{aligned}
$$

Taking $n \rightarrow \infty$, we have

$$
\begin{aligned}
1 \leq & \liminf _{n \rightarrow \infty}\left[\alpha\left(\psi\left(\omega_{\lambda}\left(f^{n-1} x_{0}, f^{n} x_{0}\right)\right)\right)+\beta\left(\psi\left(\omega_{\lambda}\left(f^{n-1} x_{0}, f^{n} x_{0}\right)\right)\right)\right. \\
& \left.+\gamma\left(\psi\left(\omega_{\lambda}\left(f^{n-1} x_{0}, f^{n} x_{0}\right)\right)\right)\right] .
\end{aligned}
$$

So, we have $\lim _{n \rightarrow \infty} \psi\left(\omega_{\lambda}\left(f^{n-1} x_{0}, f^{n} x_{0}\right)\right)=0$ and hence

$$
\lim _{n \rightarrow \infty} \omega_{\lambda}\left(f^{n-1} x_{0}, f^{n} x_{0}\right)=0,
$$

which is a contradiction of our assumption. Therefore, $\lim _{n \rightarrow \infty} \psi\left(\omega_{\lambda}\left(f^{n} x_{0}, f^{n+1} x_{0}\right)\right)=0$ and so, we have $\lim _{n \rightarrow \infty} \omega_{\lambda}\left(f^{n} x_{0}, f^{n+1} x_{0}\right)=0$. Moreover, we have $\lim _{n \rightarrow \infty} \omega_{\lambda}\left(f^{n} x_{0}, f^{n+1} x_{0}\right)=0$ for all $\lambda>0$.

Next, we show that $\left\{f^{n} x_{0}\right\}$ is a Cauchy sequence. Assume the contrary. So, there exists $\lambda_{0}, \epsilon_{0}>0$ for which we can define two subsequences $\left\{f^{m_{k}} x_{0}\right\}$ and $\left\{f^{n_{k}} x_{0}\right\}$ of the sequence $\left\{f^{n} x_{0}\right\}$ such that, for any $n_{k}>m_{k}>k, \omega_{\lambda_{0}}\left(f^{m_{k}} x_{0}, f^{n_{k}} x_{0}\right) \geq \epsilon_{0}$, but $\omega_{\lambda_{0}}\left(f^{m_{k}} x_{0}, f^{n_{k}-1} x_{0}\right)<\epsilon_{0}$. Now, since $f^{m_{k}} x_{0} \sqsubseteq f^{n_{k}} x_{0}$, we observe that

$$
\begin{aligned}
\psi\left(\epsilon_{0}\right) \leq & \psi\left(\omega_{\lambda_{0}}\left(f^{m_{k}} x_{0}, f^{n_{k}} x_{0}\right)\right) \\
\leq & \alpha\left(\psi\left(\omega_{\lambda_{0}}\left(f^{m_{k}-1} x_{0}, f^{n_{k}-1} x_{0}\right)\right)\right) \psi\left(\omega_{\lambda_{0}+\eta\left(\lambda_{0}\right)}\left(f^{m_{k}-1} x_{0}, f^{n_{k}-1} x_{0}\right)\right) \\
& +\beta\left(\psi\left(\omega_{\lambda_{0}}\left(f^{m_{k}-1} x_{0}, f^{n_{k}-1} x_{0}\right)\right)\right) \psi\left(\omega_{\lambda_{0}}\left(f^{m_{k}-1} x_{0}, f^{m_{k}} x_{0}\right)\right) \\
& +\gamma\left(\psi\left(\omega_{\lambda_{0}}\left(f^{m_{k}-1} x_{0}, f^{n_{k}-1} x_{0}\right)\right)\right) \psi\left(\omega_{\lambda_{0}}\left(f^{n_{k}-1} x_{0}, f^{n_{k}} x_{0}\right)\right) \\
\leq & \psi\left(\omega_{\eta\left(\lambda_{0}\right)}\left(f^{m_{k}-1} x_{0}, f^{m_{k}} x_{0}\right)+\omega_{\lambda_{0}}\left(f^{m_{k}} x_{0}, f^{n_{k}-1} x_{0}\right)\right) \\
& +\psi\left(\omega_{\lambda_{0}}\left(f^{m_{k}-1} x_{0}, f^{m_{k}} x_{0}\right)\right)+\psi\left(\omega_{\lambda_{0}}\left(f^{n_{k}-1} x_{0}, f^{n_{k}} x_{0}\right)\right) \\
\leq & \psi\left(\omega_{\eta\left(\lambda_{0}\right)}\left(f^{m_{k}-1} x_{0}, f^{m_{k}} x_{0}\right)+\epsilon_{0}\right)+\psi\left(\omega_{\lambda_{0}}\left(f^{m_{k}-1} x_{0}, f^{m_{k}} x_{0}\right)\right) \\
& +\psi\left(\omega_{\lambda_{0}}\left(f^{n_{k}-1} x_{0}, f^{n_{k}} x_{0}\right)\right) .
\end{aligned}
$$

Letting $k \rightarrow \infty$, we obtain that $\lim _{k \rightarrow \infty} \psi\left(\omega_{\lambda_{0}}\left(f^{m_{k}} x_{0}, f^{n_{k}} x_{0}\right)\right)=\psi\left(\epsilon_{0}\right)$. So, we have

$$
\lim _{k \rightarrow \infty} \omega_{\lambda_{0}}\left(f^{m_{k}} x_{0}, f^{n_{k}} x_{0}\right)=\epsilon_{0}
$$

Observe again that

$$
\begin{aligned}
\psi\left(\omega_{\lambda_{0}}\left(f^{m_{k}} x_{0}, f^{n_{k}} x_{0}\right)\right) \leq & \psi\left(\omega_{\lambda_{0}+\eta\left(\lambda_{0}\right)}\left(f^{m_{k}-1} x_{0}, f^{n_{k}-1} x_{0}\right)\right)+\psi\left(\omega_{\lambda_{0}}\left(f^{m_{k}-1} x_{0}, f^{m_{k}} x_{0}\right)\right) \\
& +\psi\left(\omega_{\lambda_{0}}\left(f^{n_{k}-1} x_{0}, f^{n_{k}} x_{0}\right)\right) \\
\leq & \psi\left(\omega_{\frac{\eta\left(\lambda_{0}\right)}{2}}\left(f^{m_{k}-1} x_{0}, f^{m_{k}} x_{0}\right)+\omega_{\lambda_{0}}\left(f^{m_{k}} x_{0}, f^{n_{k}} x_{0}\right)\right. \\
& \left.+\omega_{\frac{\eta\left(\lambda_{0}\right)}{2}}\left(f^{n_{k}} x_{0}, f^{n_{k}-1} x_{0}\right)\right)+\psi\left(\omega_{\lambda_{0}}\left(f^{m_{k}-1} x_{0}, f^{m_{k}} x_{0}\right)\right) \\
& +\psi\left(\omega_{\lambda_{0}}\left(f^{n_{k}-1} x_{0}, f^{n_{k}} x_{0}\right)\right) .
\end{aligned}
$$

Letting $k \rightarrow \infty$, we deduce that $\lim _{k \rightarrow \infty} \psi\left(\omega_{\lambda_{0}}\left(f^{m_{k}-1} x_{0}, f^{n_{k}-1} x_{0}\right)\right)=\psi\left(\epsilon_{0}\right)$. Similarly, we have

$$
\lim _{k \rightarrow \infty} \omega_{\lambda_{0}}\left(f^{m_{k}-1} x_{0}, f^{n_{k}-1} x_{0}\right)=\epsilon_{0}
$$


Thus, it follows that

$$
1 \leq \liminf _{k \rightarrow \infty} \alpha\left(\psi\left(\omega_{\lambda_{0}}\left(f^{m_{k}-1} x_{0}, f^{n_{k}-1} x_{0}\right)\right)\right)
$$

Therefore, we conclude that $\lim _{k \rightarrow \infty} \psi\left(\omega_{\lambda_{0}}\left(f^{m_{k}-1} x_{0}, f^{n_{k}-1} x_{0}\right)\right)=0$, which implies that

$$
\lim _{k \rightarrow \infty} \omega_{\lambda_{0}}\left(f^{m_{k}-1} x_{0}, f^{n_{k}-1} x_{0}\right)=0
$$

This is a contradiction. Therefore, it follows that $\left\{f^{n} x_{0}\right\}$ is a Cauchy sequence. Due to the completeness of $X_{\omega},\left\{f^{n} x_{0}\right\}$ converges to some point $x_{\infty} \in X_{\omega}$.

Now, we show that $x_{\infty}$ is a fixed point of $f$. Let $\lambda>0$ be arbitrary. By virtue of the condition (1.2), we consider that

$$
\psi\left(\omega_{\lambda}\left(f^{n+1} x_{0}, f x_{\infty}\right) \leq \psi\left(\omega_{\lambda}\left(f^{n} x_{0}, x_{\infty}\right)\right)+\psi\left(\omega_{\lambda}\left(f^{n} x_{0}, f^{n+1} x_{0}\right)\right)\right.
$$

Letting $n \rightarrow \infty$, we obtain that $\psi\left(\omega_{\lambda}\left(x_{\infty}, f x_{\infty}\right) \leq 0\right.$ for all $\lambda>0$. Therefore, $x_{\infty}$ is a fixed point of $f$.

Theorem 2.2. Additional to the Theorem 2.1, if $\psi$ is subadditive and the following condition holds:

$$
\begin{gathered}
\text { for any } x, y \in X_{\omega} \text {, there exists } w \in X_{\omega} \text { with } w \sqsubseteq f w \text { and } \omega_{\lambda}(w, f w)<\infty \text { for all } \lambda>0 \\
\text { such that } w \text { is comparable to both } x \text { and } y,
\end{gathered}
$$

then the fixed point in Theorem 2.1 is unique.

Proof. By Theorem 2.1, we know that $f$ has a fixed point $x_{\infty} \in X_{\omega}$. Assume that $y_{\infty} \in$ $X_{\omega}$ is also another fixed point of $f$. Thus, we can find $w \in X_{\omega}$ with $w \subseteq f w$ and comparable to both $x_{\infty}$ and $y_{\infty}$. It follows that $f^{n} w$ is comparable with both $x_{\infty}$ and $y_{\infty}$ for each $n \in \mathbb{N}$. Observe that, for any $\lambda>0$,

$$
\begin{aligned}
\psi\left(\omega_{\lambda}\left(f^{n+1} w, x_{\infty}\right)=\right. & \psi\left(\omega_{\lambda}\left(f^{n+1} w, f x_{\infty}\right)\right. \\
\leq & \alpha\left(\psi\left(\omega_{\lambda}\left(f^{n} w, x_{\infty}\right)\right)\right) \psi\left(\omega_{\lambda}\left(f^{n} w, x_{\infty}\right)\right) \\
& +\beta\left(\psi\left(\omega_{\lambda}\left(f^{n} w, x_{\infty}\right)\right)\right) \psi\left(\omega_{\lambda}\left(f^{n} w, f^{n+1} w\right)\right) \\
\leq & \alpha\left(\psi\left(\omega_{\lambda}\left(f^{n} w, x_{\infty}\right)\right)\right) \psi\left(\omega_{\lambda}\left(f^{n} w, x_{\infty}\right)\right) \\
& +\beta\left(\psi\left(\omega_{\lambda}\left(f^{n} w, x_{\infty}\right)\right)\right) \psi\left(\omega_{\lambda}\left(f^{n} w, x_{\infty}\right)\right) \\
& +\beta\left(\psi\left(\omega_{\lambda}\left(f^{n} w, x_{\infty}\right)\right)\right) \psi\left(\omega_{\lambda}\left(x_{\infty}, f^{n+1} w\right)\right) .
\end{aligned}
$$

Therefore, without loss of generality, we have

$$
\begin{aligned}
\psi\left(\omega_{\lambda}\left(f^{n+1} w, x_{\infty}\right)\right. & \leq \frac{\alpha\left(\psi\left(\omega_{\lambda}\left(f^{n} w, x_{\infty}\right)\right)\right)+\beta\left(\psi\left(\omega_{\lambda}\left(f^{n} w, x_{\infty}\right)\right)\right)}{1-\beta\left(\psi\left(\omega_{\lambda}\left(f^{n} w, x_{\infty}\right)\right)\right)} \psi\left(\omega_{\lambda}\left(f^{n} w, x_{\infty}\right)\right) \\
& \leq \psi\left(\omega_{\lambda}\left(f^{n} w, x_{\infty}\right)\right) \\
& \vdots \\
& \leq \psi\left(\omega_{\lambda}\left(w, x_{\infty}\right)\right) \\
& <\infty
\end{aligned}
$$

Therefore, $\left\{\psi\left(\omega_{\lambda}\left(f^{n} w, x_{\infty}\right)\right)\right\}$ is nonincreasing and bounded below. So, it converges to some real number $h \geq 0$. Assume that $h>0$. According to the proof of Theorem 2.1, we know that $\lim _{n \rightarrow \infty} \omega_{\lambda}\left(f^{n} w, f^{n+1} w\right)=0$ for all $\lambda>0$. Thus, letting $n \rightarrow \infty$ in the inequality (2.3), we have 


$$
1 \leq \liminf _{n \rightarrow \infty} \alpha\left(\psi\left(\omega_{\lambda}\left(f^{n} w, x_{\infty}\right)\right)\right)
$$

Thus, we have $\left\{f^{n} w\right\}$ converges to $x_{\infty}$. Similarly, we obtain that $\left\{f^{n} w\right\}$ converges also to $y_{\infty}$. Since the limit is unique, we have $x_{\infty}=y_{\infty}$. This contradicts our assumption. Therefore, the theorem is proved.

Corollary 2.3. Additional to Theorem 2.1, if $X_{\omega}$ is totally ordered, then the fixed point in Theorem 2.1 is unique.

Proof. Since $X_{\omega}$ is totally ordered, the condition (2.2) is satisfied. Thus, applying Theorem 2.2, we obtain the result.

The following two corollaries nicely broaden the results in [24] (see Theorems 3.2 and $3.6[24])$.

Corollary 2.4. Let $X_{\omega}$ be a complete modular metric space with a partial ordering $\subseteq$ and $f$ be a self-mapping on $X_{\omega}$ such that, for any $\lambda>0$, there exists $\eta(\lambda) \in(0, \lambda)$ such that

$$
\psi\left(\omega_{\lambda}(f x, f y)\right) \leq \alpha\left(\psi\left(\omega_{\lambda}(x, y)\right)\right) \psi\left(\omega_{\lambda+\eta(\lambda)}(x, y)\right),
$$

where $\alpha \in \mathcal{S}$ and $\psi \in \bar{\Psi}$. Assume also that $f$ is continuous or the condition (1.2) holds. Then $f$ has a fixed point in $X_{\omega}$. Moreover, if the condition (2.2) is satisfied, the fixed point is unique.

Proof. Since $\alpha \in \mathcal{S}$, we have $(\alpha, \theta, \theta) \in \mathcal{S}_{3}$. Thus, apply Theorems 2.1 and 2.2, we have the conclusion.

Corollary 2.5. Let $X_{\omega}$ be a complete modular metric space with a partial ordering 드 and $f$ be a self-mapping on $X_{\omega}$ such that, for any $\lambda>0$, there exist $\zeta(\lambda), \mu(\lambda) \in(0, \lambda)$ such that

$$
\psi\left(\omega_{\lambda}(f x, f y)\right) \leq \beta\left(\psi\left(\omega_{\lambda}(x, y)\right)\right) \psi\left(\omega_{\lambda}(x, f x)\right)+\gamma\left(\psi\left(\omega_{\lambda}(x, y)\right)\right) \psi\left(\omega_{\lambda}(y, f y)\right),
$$

where $\psi \in \bar{\Psi}$ and $(\beta, \gamma) \in \mathcal{S}_{2}$ with $\max \left\{\sup _{t \geq 0} \beta(t), \sup _{t \geq 0} \gamma(t)\right\}<1$. Assume also that $f$ is continuous or that the condition (1.2) holds. Then $f$ has a fixed point in $X_{\omega}$. Moreover, if the condition (2.2) is satisfied, the fixed point is unique.

Proof. Since $(\beta, \gamma) \in \mathcal{S}_{2}$, we have $(\theta, \beta, \gamma) \in \mathcal{S}_{3}$. Thus, apply Theorems 2.1 and 2.2, we have the conclusion.

\section{Applications}

In this section, we give an application of our theorems to establish the existence and uniqueness of a solution to a nonhomogeneous linear parabolic partial differential equation satisfying a given initial condition.

Consider the following initial value problem

$$
\begin{cases}u_{t}(x, t)=u_{x x}(x, t)+F\left(x, t, u(x, t), u_{x}(x, t)\right), & -\infty<x<\infty, 0<t \leq T \\ u(x, 0)=\varphi(x) \geq 0, & -\infty<x<\infty\end{cases}
$$

where we assume $\phi$ to be continuously differentiable such that $\phi$ and $\phi$ ' are bounded and $F$ is continuous.

By a solution of the system (3.1), we meant a function $u \equiv u(x, t)$ defined on $\mathbb{R} \times I$, where $I:=[0, T]$, satisfying the following conditions: 
(a) $u, u_{t}, u_{x}, u_{x x} \in C(\mathbb{R} \times I)$.

(b) $u$ and $u_{x}$ are bounded in $\mathbb{R} \times I$.

(c) $u_{t}(x, t)=u_{x x}(x, t)+F\left(x, t, u(x, t), u_{x}(x, t)\right)$ for all $(x, t) \in \mathbb{R} \times I$.

(d) $u(x, 0)=\phi(x)$ for all $x \in \mathbb{R}$.

Now, we consider the following space:

$$
\Omega:=\left\{u(x, t): u, u_{x} \in C(\mathbb{R} \times I) \text { and }\|u\|<\infty\right\},
$$

where

$$
\|u\|:=\sup _{x \in \mathbb{R}, t \in I}|u(x, t)|+\sup _{x \in \mathbb{R}, t \in I}\left|u_{x}(x, t)\right| .
$$

Obviously, the function $\omega: \mathbb{R}^{+} \times \Omega \times \Omega \rightarrow \mathbb{R}_{+}$given by

$$
\omega_{\lambda}(x, y):=\frac{1}{1+\lambda}\|u-v\|
$$

is a metric modular on $\Omega$. Clearly, the set $\Omega_{\omega}$ is a complete modular metric space independent of generators. Define a partial ordering $\subseteq$ on $\Omega_{\omega}$ by

$$
u, v \in \Omega_{\omega}, u \sqsubseteq v \Leftrightarrow u(x, t) \leq v(x, t) \text { and } u_{x}(x, t) \leq v_{x}(x, t) \text { at each }(x, t) \in \mathbb{R} \times I .
$$

Taking a nondecreasing sequence $\left\{u_{n}\right\}$ in $\Omega_{\omega}$ converging to $u \in \Omega_{\omega}$. For any $(x, t) \in$ $\mathbb{R} \times I$,

we have

$$
u_{1}(x, t) \leq u_{2}(x, t) \leq \cdots \leq u_{n}(x, t) \leq \cdots
$$

and

$$
\left(u_{1}\right)_{x}(x, t) \leq\left(u_{2}\right)_{x}(x, t) \leq \cdots \leq\left(u_{n}\right)_{x}(x, t) \leq \cdots .
$$

Since the sequences $\left\{u_{n}(x, t)\right\}$ and $\left\{\left(u_{n}\right)_{x}(x, t)\right\}$ converges to $u(x, t)$ and $u_{x}(x, t)$, respectively, it follows that, for any $(x, t) \in \mathbb{R} \times I$,

$$
u_{n}(x, t) \leq u(x, t) \text { and }\left(u_{n}\right)_{x}(x, t) \leq u_{x}(x, t)
$$

for all $n \geq 1$. Therefore, $u_{n} \sqsubseteq u$ for all $n \geq 1$. So, the space $\Omega_{\omega}$ satisfies the condition (1.2).

Theorem 3.1. Consider the problem (3.1) and assume the following:

(1) For any $c>0$ with $|s|<c$ and $|p|<c$, the function $F(x, t, s, p)$ is uniformly Hölder continuous in $X$ and $t$ for each compact subset of $\mathbb{R} \times I$.

(2) There exists a constant $c_{F} \leq\left(T+2 \pi^{-\frac{1}{2}} \frac{1}{2}\right)^{-1}$ such that, for any $\lambda>0$, there exists $\eta(\lambda) \in(0, \lambda)$ such that

$$
\begin{aligned}
0 & \leq \frac{1}{1+\lambda}\left[F\left(x, t, s_{2}, p_{2}\right)-F\left(x, t, s_{1}, p_{1}\right)\right] \\
& \leq c_{F}\left[\frac{1}{1+\lambda+\eta(\lambda)} \rho\left(\Xi\left(\frac{s_{2}-s_{1}+p_{2}-p_{1}}{1+\lambda}\right)\right)+\frac{1}{1+\lambda} \sigma\left(\Xi\left(\frac{s_{2}-s_{1}+p_{2}-p_{1}}{1+\lambda}\right)\right)\right]
\end{aligned}
$$


for all $\left(s_{1}, p_{1}\right),\left(s_{2}, p_{2}\right) \in \mathbb{R} \times \mathbb{R}$ with $s_{1} \leq s_{2}$ and $p_{1} \leq p_{2}$, where $\Xi \in \bar{\Psi}$ is sublinear with $\Xi(x) \leq t$ and $\rho$, $\sigma$ are nondecreasing functions on $\mathbb{R}_{+}$such that $\rho(t)<(1-k) t$ and $\sigma(t)<(1-k) k t$ for all $t>0$ and for some fixed $k \in(0,1)$.

(3) The two functions $\Gamma, \Upsilon: \mathbb{R}_{+} \rightarrow[0,1)$ given by

$$
\Gamma(t)=\left\{\begin{array}{cl}
0 & \text { if } t=0, \\
\frac{\rho(t)}{(1-k) t} & \text { if } t>0,
\end{array} \quad \Upsilon(t)=\left\{\begin{array}{cl}
0 & \text { if } t=0, \\
\frac{\sigma(t)}{(1-k) t} & \text { if } t>0,
\end{array}\right.\right.
$$

are such that $(\Gamma, \Upsilon, \Upsilon) \in \mathcal{S}_{3}, \Gamma+2 \Upsilon<1$.

(4) $F(x, t, s, 0) \geq \frac{s}{\int_{0}^{t} \int_{\infty}^{\infty} k(x-\xi, t-\tau) d \xi d \tau}$ for all $s \geq 0$.

(5) $F$ is bounded for bounded $s$ and $p$.

Then, the existence and uniqueness of the solution of the system (3.1) is affirmative.

It is essential to note that the problem (3.1) is equivalent (under the assumption of Theorem 3.1) to the integral equation:

$$
u(x, t)=\int_{-\infty}^{\infty} k(x-\xi, t) \varphi(\xi) d \xi+\int_{0}^{t} \int_{-\infty}^{\infty} k(x-\xi, t-\tau) F\left(\xi, \tau, u(\xi, \tau), u_{x}(\xi, \tau)\right) d \xi d \tau
$$

for all $x \in \mathbb{R}$ and $0<t \leq T$, where

$$
k(x, t):=\frac{1}{\sqrt{4 \pi t}} e^{-\frac{x^{2}}{4 t}}
$$

for all $x \in \mathbb{R}$ and $t>0$. The system (3.1) possesses a unique solution if and only if the equation (3.2) possesses a unique solution $u$ such that $u$ and $u_{x}$ are both continuous and bounded for all $x \in \mathbb{R}$ and $0<t \leq T$.

Define a mapping $\Lambda: \Omega_{\omega} \rightarrow \Omega_{\omega}$ by

$$
(\Lambda u)(x, t):=\int_{-\infty}^{\infty} k(x-\xi, t) \varphi(\xi) d \xi+\int_{0}^{t} \int_{-\infty}^{\infty} k(x-\xi, t-\tau) F\left(\xi, \tau, u(\xi, \tau), u_{x}(\xi, \tau)\right) d \xi d \tau
$$

for all $(x, t) \mathbb{R} \times I$. Then the problem of finding the solution to the equation (3.2) is equivalent to the problem of finding the fixed point of $\Lambda$.

Proof (Theorem 3.1). It is easy to see that the mapping $\Lambda$ is nondecreasing by the definition. Let $u, v \in \Omega_{\omega}$ with $u \subseteq v$. Suppose that $u \neq v$. Besides, we have

$$
\begin{aligned}
\frac{1}{1+\lambda} \mid & (\Lambda v)(x, t)-(\Lambda u)(x, t) \mid \\
\leq & \frac{1}{1+\lambda} \int_{0}^{t} \int_{-\infty}^{\infty} k(x-\xi, t-\tau)\left|F\left(\xi, \tau, v(\xi, \tau), v_{x}(\xi, \tau)\right)-F\left(\xi, \tau, u(\xi, \tau), u_{x}(\xi, \tau)\right)\right| d \xi d \tau \\
\leq & \int_{0}^{t} \int_{-\infty}^{\infty} k(x-\xi, t-\tau) c_{F}\left[\frac{1}{1+\lambda+\eta(\lambda)} \rho\left(\Xi\left(\frac{1}{1+\lambda}\left(v(\xi, \tau)-u(\xi, \tau)+v_{x}(\xi, \tau)-u_{x}(\xi, \tau)\right)\right)\right)\right. \\
& \left.+\frac{1}{1+\lambda} \sigma\left(\Xi\left(\frac{1}{1+\lambda}\left(v(\xi, \tau)-u(\xi, \tau)+v_{x}(\xi, \tau)-u_{x}(\xi, \tau)\right)\right)\right)\right] d \xi d \tau \\
\leq & c_{F}\left[\frac{1}{1+\lambda+\eta(\lambda)} \rho\left(\Xi\left(\omega_{\lambda}(u, v)\right)\right)+\frac{1}{1+\lambda} \sigma\left(\Xi\left(\omega_{\lambda}(u, v)\right)\right)\right] \int_{0}^{t} \int_{-\infty}^{\infty} k(x-\xi, t-\tau) d \xi d \tau \\
\leq & c_{F}\left[\frac{1}{1+\lambda+\eta(\lambda)} \rho\left(\Xi\left(\omega_{\lambda}(u, v)\right)\right)+\frac{1}{1+\lambda} \sigma\left(\Xi\left(\omega_{\lambda}(u, v)\right)\right)\right] T .
\end{aligned}
$$


Similarly, we have

$$
\begin{aligned}
& \frac{1}{1+\lambda}\left|(\Lambda v)_{x}(x, t)-(\Lambda u)_{x}(x, t)\right| \\
& \quad \leq c_{F}\left[\frac{1}{1+\lambda+\eta(\lambda)} \rho\left(\Xi\left(\omega_{\lambda}(u, v)\right)\right)+\frac{1}{1+\lambda} \sigma\left(\Xi\left(\omega_{\lambda}(u, v)\right)\right)\right] \int_{0}^{t} \int_{-\infty}^{\infty}\left|k_{x}(x-\xi, t-\tau)\right| d \xi d \tau \\
& \quad \leq 2 \pi^{-\frac{1}{2}} T^{-\frac{1}{2}} c_{F}\left[\frac{1}{1+\lambda+\eta(\lambda)} \rho\left(\Xi\left(\omega_{\lambda}(u, v)\right)\right)+\frac{1}{1+\lambda} \sigma\left(\Xi\left(\omega_{\lambda}(u, v)\right)\right)\right] .
\end{aligned}
$$

Note that by (2), we have $\sup _{t \geq 0} \Upsilon(t) \leq k<1$. Together with (3.3) and (3.4), we obtain

$$
\begin{aligned}
\omega_{\lambda}(\Lambda u, \Lambda v) \leq & \left(T+2 \pi^{-\frac{1}{2}} T^{\frac{1}{2}}\right) c_{F}\left[\frac{1}{1+\lambda+\eta(\lambda)} \rho\left(\Xi\left(\omega_{\lambda}(u, v)\right)\right)+\frac{1}{1+\lambda} \sigma\left(\Xi\left(\omega_{\lambda}(u, v)\right)\right)\right] \\
\leq & \frac{1}{1+\lambda+\eta(\lambda)} \rho\left(\Xi\left(\omega_{\lambda}(u, v)\right)\right)+\frac{1}{1+\lambda} \sigma\left(\Xi\left(\omega_{\lambda}(u, v)\right)\right) \\
= & \frac{\|u-v\|}{1+\lambda+\eta(\lambda)} \frac{\rho\left(\Xi\left(\omega_{\lambda}(u, v)\right)\right)}{\|u-v\|}+\frac{\|u-v\| \mid}{1+\lambda} \frac{\sigma\left(\Xi\left(\omega_{\lambda}(u, v)\right)\right)}{\|u-v\|} \\
\leq & \omega_{\lambda+\eta(\lambda)}(u, v) \frac{\rho\left(\Xi\left(\omega_{\lambda}(u, v)\right)\right)}{\omega_{\lambda}(u, v)}+\omega_{\lambda}(u, \Lambda u) \frac{\sigma\left(\Xi\left(\omega_{\lambda}(u, v)\right)\right)}{\left.\omega_{\lambda}(u, v)\right)} \\
& +\omega_{\lambda}(v, \Lambda v) \frac{\sigma\left(\Xi\left(\omega_{\lambda}(u, v)\right)\right)}{\omega_{\lambda}(u, v)}+\omega_{\lambda}(\Lambda u, \Lambda v) \frac{\sigma\left(\Xi\left(\omega_{\lambda}(u, v)\right)\right)}{\omega_{\lambda}(u, v)} \\
\leq & \omega_{\lambda+\eta(\lambda)}(u, v) \frac{\rho\left(\Xi\left(\omega_{\lambda}(u, v)\right)\right)}{\omega_{\lambda}(u, v)}+\omega_{\lambda}(u, \Lambda u) \frac{\sigma\left(\Xi\left(\omega_{\lambda}(u, v)\right)\right)}{\omega_{\lambda}(u, v)} \\
& +\omega_{\lambda}(v, \Lambda v) \frac{\sigma\left(\Xi\left(\omega_{\lambda}(u, v)\right)\right)}{\omega_{\lambda}(u, v)}+\omega_{\lambda}(\Lambda u, \Lambda v) \Upsilon\left(\omega_{\lambda}(u, v)\right) \\
\leq & \omega_{\lambda+\eta(\lambda)}(u, v) \frac{\rho\left(\Xi\left(\omega_{\lambda}(u, v)\right)\right)}{\omega_{\lambda}(u, v)}+\omega_{\lambda}(u, \Lambda u) \frac{\sigma\left(\Xi\left(\omega_{\lambda}(u, v)\right)\right)}{\omega_{\lambda}(u, v)} \\
& +\omega_{\lambda}(v, \Lambda v) \frac{\sigma\left(\Xi\left(\omega_{\lambda}(u, v)\right)\right)}{\omega_{\lambda}(u, v)}+k \omega_{\lambda}(\Lambda u, \Lambda v) \\
\leq & \omega_{\lambda+\eta(\lambda)}(u, v) \frac{\rho\left(\Xi\left(\omega_{\lambda}(u, v)\right)\right)}{\Xi\left(\omega_{\lambda}(u, v)\right)}+\omega_{\lambda}(u, \Lambda u) \frac{\sigma\left(\Xi\left(\omega_{\lambda}(u, v)\right)\right)}{\Xi\left(\omega_{\lambda}(u, v)\right)} \\
& +\omega_{\lambda}(v, \Lambda v) \frac{\sigma\left(\Xi\left(\omega_{\lambda}(u, v)\right)\right)}{\Xi\left(\omega_{\lambda}(u, v)\right)}+k \omega_{\lambda}(\Lambda u, \Lambda v) .
\end{aligned}
$$

Further, we obtain

$$
\begin{aligned}
\omega_{\lambda}(\Lambda u, \Lambda v) \leq & \omega_{\lambda+\eta(\lambda)}(u, v) \Gamma\left(\Xi\left(\omega_{\lambda}(u, v)\right)\right)+\omega_{\lambda+\zeta(\lambda)}(u, \Lambda u) \Upsilon\left(\Xi\left(\omega_{\lambda}(u, v)\right)\right) \\
& +\omega_{\lambda+\mu(\lambda)}(v, \Lambda v) \Upsilon\left(\Xi\left(\omega_{\lambda}(u, v)\right)\right)
\end{aligned}
$$

Moreover, since $\xi$ is a sublinear (real) function in the class $\Psi$, it follows that

$$
\begin{aligned}
\Xi\left(\omega_{\lambda}(\Lambda u, \Lambda v)\right) \leq & \Xi\left(\omega_{\lambda+\eta(\lambda)}(u, v)\right) \Gamma\left(\Xi\left(\omega_{\lambda}(u, v)\right)\right)+\Xi\left(\omega_{\lambda+\zeta(\lambda)}(u, \Lambda u)\right) \Upsilon\left(\Xi\left(\omega_{\lambda}(u, v)\right)\right) \\
& +\Xi\left(\omega_{\lambda+\mu(\lambda)}(v, \Lambda v)\right) \Upsilon\left(\Xi\left(\omega_{\lambda}(u, v)\right)\right) .
\end{aligned}
$$

For the case $u=v$, it is obvious that the above inequality is satisfied. Thus, we now have the inequality (2.1) holds for any comparable $u, v \in \Omega_{\omega}$.

Note that any constant functions are contained in $\Omega$. Now, for any $u, v \in \Omega_{\omega}$, we may choose a constant function $w \in \Omega_{\omega}$ for which $u, v \sqsubseteq w$ and $\theta \subseteq w$. Consequently, we have $w(x, t)=\|w\|$ for all $(x, t) \in \mathbb{R} \times I$. Also, observe that this $w$ attains the following: 


$$
\begin{aligned}
(\Lambda w)(x, t) & =\int_{-\infty}^{\infty} k(x-\xi, t) \varphi(\xi) d \xi+\int_{0}^{t} \int_{-\infty}^{\infty} k(x-\xi, t-\tau) F(\xi, \tau,\|w\|, 0) d \xi d \tau \\
& \geq \int_{-\infty}^{\infty} k(x-\xi, t) \varphi(\xi) d \xi+\|w\| \\
& \geq\|w\| \\
& =w(x, t) .
\end{aligned}
$$

Hence, the condition (2.2) is satisfied. Therefore, by applying the Theorems 2.1 and 2.2 , the result follows. This completes the proof.

\section{Acknowledgements}

The authors were supported by the Higher Education Research Promotion and National Research University Project of Thailand, Office of the Higher Education Commission (NRU-CSEC No.55000613). This research was partially finished at Department of Mathematics Education and the RINS, Gyeongsang National University, Chinju, Korea, while the first and third authors visit here. Also, the second author was supported by the Basic Science Research Program through the National Research Foundation of Korea (NRF) funded by the Ministry of Education, Science, and Technology (Grant no. 2011-0021821). Furthermore, the authors are grateful for the reviewers for the careful reading of the article and for the suggestions which improved the quality of this work.

\section{Author details}

${ }^{1}$ Department of Mathematics, Faculty of Science, King Mongkut's University of Technology Thonburi, Bangkok 10140, Thailand ${ }^{2}$ Department of Mathematics Education and the RINS, Gyeongsang National University, Chinju 660-701, Korea

\section{Authors' contributions}

All authors read and approved the final manuscript.

\section{Competing interests}

The authors declare that they have no competing interests.

Received: 3 May 2012 Accepted: 20 June 2012 Published: 20 June 2012

\section{References}

1. Golubović, Z, Kadelburg, Z, Radenović, S: Coupled coincidence points of mappings in ordered partial metric spaces. Abstr Appl Anal 2012, 18 (2012). Article ID 192581

2. Khamsi, MA, Latif, A, Al-Sulami, H: KKM and Ky Fan theorems in modular function spaces. Fixed Point Theory Appl. 2011, 57 (2011). doi:10.1186/1687-1812-2011-57

3. Khamsi, MA: Quasicontraction mappings in modular spaces without $\Delta_{2}$-condition. Fixed Point Theory Appl 2008, 6 (2008). Article ID 916187

4. Kuaket, K, Kumam, P: Fixed points of asymptotic pointwise contractions in modular spaces. Appl Math Lett. 24 1795-1798 (2011). doi:10.1016/j.aml.2011.04.035

5. Amini-harandini, A: Fixed point theory for generalized quasicontraction maps in vector modular spaces. Comput Math Appl. 61, 1891-1897 (2011). doi:10.1016/j.camwa.2011.02.021

6. Aydi, H, Nashine, HK, Samet, B, Yazidi, H: Coincidence and common fixed point results in partially ordered cone metric spaces and applications to integral equations. Nonlinear Anal. 74(17), 6814-6825 (2011). doi:10.1016/j.na.2011.07.006

7. Aydi, $\mathrm{H}$ : Common fixed point results for mappings satisfying $(\psi, \nabla)$-weak contractions in ordered partial metric spaces. Int J Math Stat. 12(2), 53-64 (2012)

8. Sintunavarat, W, Kumam, P: Weak condition for generalized multi-valued $(f, a, \beta)$-weak contraction mappings. Appl Math Lett. 24, 460-465 (2011). doi:10.1016/j.aml.2010.10.042

9. Sintunavarat, W, Cho, YJ, Kumam, P: Common fixed point theorems for c-distance in ordered cone metric spaces Comput Math Appl. 62, 1969-1978 (2011). doi:10.1016/j.camwa.2011.06.040

10. Mongkolkeha, C, Kumam, P: Fixed point theorems for generalized asymptotic pointwise $\rho$-contraction mappings involving orbits in modular function spaces. Appl Math Lett.2012http://dx.doi.org/10.1016/j.aml.2011.11.027

11. Geraghty, M: On contractive mappings. Proc Am Math Soc. 40, 604-608 (1973). doi:10.1090/S0002-9939-1973-0334176-5

12. Amini-Harandi, A, Emani, H: A fixed point theorem for contraction type maps in partially ordered metric spaces and application to ordinary differential equations. Nonlinear Anal. 72, 2238-2242 (2010). doi:10.1016/j.na.2009.10.023

13. Eshaghi Gordji, M, Ramezani, M, Cho, YJ, Pirbavafa, S: A generalization of Geraghty's theorem in partially ordered metric spaces and application to ordinary differential equations. Fixed Point Theory Appl. 2012, 74 (2012)

14. Chistyakov, W: Modular metric spaces, I: basic concepts. Nonlinear Anal. 72, 1-14 (2010). doi:10.1016/j.na.2009.04.057

15. Nakano, H: Modulared Semi-Ordered Linear Spaces. In Tokyo Mathematics Book Series, vol. 1,Maruzen Co., Tokyo (1950)

16. Nakano, H: Topology and Linear Topological Spaces. In Tokyo Mathematics Book Series, vol. 3,Maruzen Co., Tokyo (1951)

17. Kumam, P: Some geometric properties and fixed point theorem in modular spaces. In: Garcia Falset J, Fuster L, Sims B (eds.) Fixed Point Theorem and its Applications. pp. 173-188. Yokohama Publishers, Yokohama (2004) 
18. Kumam, P: Fixed point theorems for nonexpansive mappings in, odular spaces. Arch Math (BRONO). 40(4), 345-353 (2004)

19. Kumam, P: On uniform opial condition and uniform Kadec-Klee property in modular spaces. J Interdiscip Math. 8(3), 377-385 (2005)

20. Kumam, P: On nonsquare and Jordan-Von Neumann constants of modular spaces. Southeast Asian Bull Math. 30(1) 67-77 (2006)

21. Mongkolkeha, C, Kumam, P: Common fixed point for generallized weak contraction mapping in modular spaces. Sci Math Japon117-127. e-2012 (online)

22. Cho, YJ, Saadati, R, Sadeghi, G: Quasi-contractive mappings in modular metric spaces. J Appl Math 2012, 5 (2012). Article ID 907951

23. Chaipunya, $\mathrm{P}$, Mongkolkeha, C, Sintunavarat, W, Kumam, P: Fixed-point theorems for multivalued mappings in modular metric spaces. Abstr Appl Anal 2012, 14 (2012). Article ID 503504

24. Mongkolkeha, C, Sintunavarat, W, Kumam, P: Fixed point theorems for contraction mappings in modular metric spaces. Fixed Point Theory Appl. 2011, 93 (2011). doi:10.1186/1687-1812-2011-93

doi:10.1186/1687-1847-2012-83

Cite this article as: Chaipunya et al:: Geraghty-type theorems in modular metric spaces with an application to partial differential equation. Advances in Difference Equations 2012 2012:83.

\section{Submit your manuscript to a SpringerOpen ${ }^{\circ}$} journal and benefit from:

- Convenient online submission

- Rigorous peer review

- Immediate publication on acceptance

- Open access: articles freely available online

- High visibility within the field

- Retaining the copyright to your article

Submit your next manuscript at $\boldsymbol{s p r i n g e r o p e n . c o m ~}$ 